\title{
Bethesda Unit per Milliliter
}

National Cancer Institute

\section{Source}

National Cancer Institute. Bethesda Unit per Millilter. NCI Thesaurus. Code 6117967.

A unit of measurement for blood coagulation inhibitor activity, expressed as a Bethesda

Unit per unit of volume equal to one milliliter. 